\title{
ESTUDIO DE PROPIEDADES PSICOMÉTRICAS DE UN CUESTIONARIO DE AFRONTAMIENTO, APOYO SOCIAL Y CONDUCTA ALIMENTARIA EN PERSONAS CON DIABETES E HIPERTENSIÓN \\ STUDY OF PSYCHOMETRIC PROPERTIES OF A COPING, SOCIAL SUPPORT AND FOOD BEHAVIOR QUESTIONNAIRE IN PERSONS WITH DIABETES AND HYPERTENSION
}

Recibido: 22 de junio de 2017 | Aceptado: 25 de julio de 2017

DOI: $10.22199 / 507187475.2017 .0002 .00004$

\section{PAMELA FRANCO 1 ; XAVIER URTUBEY 2 ; ANA MARÍA GALLARDO 2}

1, 2 ACCUHEALTH, Santiago de Chile, Chile

\begin{abstract}
RESUMEN
INTRODUCCIÓN: La adaptación a la enfermedad crónica es un proceso complejo que se ve influenciado por aspectos psicosociales. Considerando la corta duración las consultas médicas, sería beneficioso contar con instrumentos que evalúen estos aspectos de manera rápida y simple, permitiendo identificar posibles focos de intervención. OBJETIVO: El objetivo de este estudio fue analizar las propiedades psicométricas de un cuestionario que evalúa afrontamiento de la enfermedad crónica, apoyo social y conducta alimentaria en personas con diabetes y/o hipertensión. METODO: Para la construcción del cuestionario se generó un conjunto de ítems, tanto creados por los autores como obtenidos a través de una revisión de cuestionarios existentes, de los cuales se seleccionaron 25. Participaron 216 individuos con diabetes y/o hipertensión, que ingresaban a un programa de monitoreo a distancia de su patología. Se realizaron análisis paralelo para el número de factores, análisis factorial de ejes principales, de confiabilidad y de regresión lineal múltiple y logística con datos informados por los pacientes (por ejemplo: $\mathrm{HbAlc}$ ) y observables a través del programa de telemonitoreo (datos bioquímicos de glicemia y/o presión arterial y adherencia al programa). RESULTADOS: Se extrajeron 5 factores que se denominaron: afrontamiento activo, evitativo y de resignación pasiva, sobrealimentación y apoyo social. Los factores presentaron una fiabilidad de .54 a .91 . Las estrategias de afrontamiento se asociaron a adherencia a los tratamientos y gravedad de la patología. CONCLUSIONES: Los resultados sugieren que el cuestionario posee propiedades psicométricas aceptables, permitiendo pesquisar aspectos psicosociales que influyen en el manejo y pronóstico de la patología.
\end{abstract}

PALABRAS CLAVE: Diabetes, hipertensión, cuestionario, aspectos psicológicos, afrontamiento.

\begin{abstract}
INTRODUCTION: Adapting to chronic disease is a complex process influenced by psychosocial factors. Considering the rather limited time of medical consultations, it would be beneficial to have instruments that evaluate these psychosocial factors quickly and easily, allowing the identification of potential focuses of intervention. OBJECTIVE: To analyze the psychometric properties of a questionnaire that assesses coping with chronic illness, social support and eating behavior in people with diabetes and/or hypertension. METHODS: The questionnaire is composed of a set of 25 items generated by both the authors and existing questionnaires. The subjects for this study are 216 diabetes and/or hypertension patients, who joined a remote monitoring program for these pathologies. The analysis considered parallel analyzes for the number of factors, factorial analysis (principal axis), reliability and multiple linear or logistic regression with data reported by the patients (for example, HbAlc) as well as and observable data obtained with a telemonitoring program (biochemical glycemia data and/or blood pressure and adherence to the program). RESULTS: Five factors were identified: active, avoidance and passive resignation coping, overfeeding and social support. Factors presented a reliability of .54 to .91 . Coping strategies were associated with adherence to treatments and pathology severity. CONCLUSIONS: Results suggest the questionnaire has acceptable psychometrical properties, allowing the identification of psychosocial aspects that influence the management and prognosis of the pathology.
\end{abstract}

KEY WORDS: Diabetes, hypertension, questionnaire, psychological aspects, coping.

1.Accuhealth, Isidora Goyenechea 2800, Piso 37, Santiago, Chile. Correo electrónico: xurtubey@accuhealth.cl. Teléfono: (+56) 23692375.

2. Pontificia Universidad Católica de Chile, Departamento de Psicología, Av. Libertador Bernardo 0\&\#39;Higgins 328, Santiago, Chile. 
Las enfermedades crónicas son una de las principales causas de defunción y discapacidad a nivel mundial (OMS, 2014). En Chile, la hipertensión arterial (HTA) es la enfermedad crónica más común, con una prevalencia de $27 \%$ en personas sobre los 15 años de edad, aumentando a $75 \%$ en personas sobre los 65 años. Por otro lado, Chile tiene la mayor prevalencia de diabetes mellitus (DM) tipo 2 en América del Sur (FID, 2015), con un $9.4 \%$ de prevalencia en la población total y un $26 \%$ en la población mayor de 65 años (MINSAL, 2010). Ambos diagnósticos tienden a co-ocurrir en el mismo individuo, lo que sugiere la existencia de factores predisponentes comunes (ver revisión de Cheung \& Li, 2012). Al momento del diagnóstico de DM tipo 2, 30-50\% presentan HTA, número que crece a $70 \%$ cuando desarrollan nefropatía (Solís et al., 2008).

En Chile, estudios muestran que del total de las personas diagnosticadas con HTA, sólo un cuarto presenta su presión arterial controlada y de aquellos con DM, sólo un tercio presenta un adecuado control metabólico (MINSAL, 2010). Finalmente, de quienes presentan ambos diagnósticos de DM y HTA, sólo el $1,7 \%$ cumple con los objetivos terapéuticos en glicemia, presión arterial y colesterol (MINSAL, 2011).

La adaptación a la enfermedad crónica es un proceso complejo, dinámico y heterogéneo, que se ve influenciado por aspectos sociodemográficos y psicosociales (Stanton, Revenson \& Tennen, 2007). A continuación, se presentan tres aspectos psicosociales que juegan un rol en este proceso: las estrategias de afrontamiento y el apoyo social percibido, constructos altamente estudiados en su relación con la adaptación y manejo de la enfermedad crónica (Manne, 2001; Gallant, 2003); y el comportamiento alimentario asociado a aspectos psicológicos, un elemento menos reconocido en la literatura (Yannakoulia, 2006; Spoor, Bekker, Van Strien, \& van Heck, 2007).

\section{Estrategias de Afrontamiento}

Las estrategias de afrontamiento consisten en intentos cognitivos o comportamentales por manejar demandas situacionales específicas que son estimadas como difíciles (Lazarus \& Folkman, 1984). Según Feifel y colegas (1987), existen tres categorías de estrategias de afrontamiento que las personas con patologías médicas utilizarían al lidiar con su enfermedad: activo, evitativo y de resignación pasiva. El afrontamiento activo es una forma positiva de afrontar que tiende a reducir el estrés asociado a enfermedades mediante acciones resolutivas que permiten lograr el control de la enfermedad y su estabilidad (Duangdao \& Roesch, 2008). La evitación 0 la resignación pasiva son formas negativas de afrontar que tienden a incrementar el estrés al impedir 0 interferir con un afrontamiento activo (Aldwin, 2007). En la evitación hay una minimización del problema y el diagnostico no se vive como una amenaza, por lo que no se realizan estrategias dirigidas al problema. En cambio, en la resignación pasiva hay una aceptación pasiva del problema, donde pueden 0 no estar presente estrategias dirigidas al problema, pero el diagnostico se ve como una amenaza o pérdida y se percibe una ausencia de control sobre el pronóstico de la enfermedad (Moorey \& Greer, 1989).

Se ha observado que individuos con DM que utilizan en mayor medida estrategias evitativas y/o de resignación pasiva presentan una peor adherencia a la dieta (Turan, Osar, Turan, Damci, \& Ilkova, 2002), chequean menos su glicemia, presentan un peor control glicémico (Iturralde, WeissbergBenchell \& Hood, 2016), y pueden experimentar estrés asociado a la diabetes y/o sintomatología depresiva (Shah, Gupchup, Borrego, Raisch \& Knapp, 2012). En individuos con HTA un mayor uso de estrategias de afrontamiento evitativas se ha asociado a un aumento de la mortalidad por cardiopatía isquémica (Svensson et al, 2016). 
Apoyo Social

La mayoría de las tareas adaptativas en la enfermedad crónica requieren de ayuda por parte de otros, incluyendo contención emocional (apoyo emocional) y ayuda práctica (apoyo instrumental) (Stanton et al, 2007). El apoyo social afecta el proceso de adaptación a la enfermedad a través de vías fisiológicas, emocionales y cognitivas (ver Willis \& Fegan, 2001). Puede ayudar a quienes lo reciben a utilizar estrategias de afrontamiento efectivas al ofrecer una mejor comprensión del problema y aumentando la motivación a tomar acciones, contribuye a incentivar el cuidado de la salud o minimizar los comportamientos de riesgo, y disminuye la reactividad fisiológica al estrés (Stanton et al, 2007).

Pacientes con DM que reportan percibir bajos niveles de apoyo social presentan un peor estado de salud/bienestar general (Westaway, Seager, Rheeder, \& Van Zyl, 2005) y una baja adherencia a los tratamientos y conductas de autocuidado (Coffman, 2008; Gallant, 2003). Con respecto a la HTA, estudios han mostrado que percibir una baja red de apoyo también estaría asociado a una peor adherencia a los tratamientos, en especial a los medicamentos y a la dieta (Marín \& Rodríguez, 2001), y a un incremento de la presión arterial (Menéndez et al., 2003). Además, el apoyo social parece mediar el efecto del estrés agudo en la presión arterial, especialmente el apoyo de tipo instrumental (Bowen et al, 2014).

\section{Conducta Alimentaria}

La dieta es una parte integral del manejo de la DM y HTA, con la cual se busca obtener óptimos resultados metabólicos y hemodinámicos, la prevención y tratamiento de complicaciones y un mejoramiento general de la salud (Franz et al, 2002; Nguyen, Odelola, Rangaswami, \& Amanullah, 2013). Sin embargo, estudios reportan una muy baja adherencia al régimen (Peyrot et al, 2005). Los individuos refieren presentar dificultades para seguir las indicaciones (Ary, Toobert, Wilson, \& Glasgow, 1986) y aquellos que adhieren a menudo reportan sentirse deprivados y tener que lidiar con fuertes deseos por comer ciertos alimentos (Hall, Joseph, \& Schwartez-Barcott, 2003; Yannakoulia, 2006). Por consecuencia, se ha observado que la restricción crónica en la dieta puede llevar a episodios de sobrealimentación (Siquiera, Dos Santos, Zanetti, \& Ferronato, 2007).

Se distinguen dos tipos de sobrealimentación: la alimentación emocional y la alimentación externa 0 impulsiva. La alimentación emocional consiste en comer en respuesta a emociones negativas con el fin de aliviar el estrés, sin considerar las señales fisiológicas internas como el hambre y la saciedad (Spoor et al, 2007). La alimentación externa se refiere al comer en respuesta de estímulos relacionados con comida (ver, oler o pensar en ciertos alimentos), sin considerar las señales fisiológicas internas. Ambas tienden a coocurrir y se han asociado con cuadros depresivos (Ouwens, Tatjana van Strien, \& van Leeuwe., 2009). Estos tipos de alimentación puede que se encuentren en el individuo previo al diagnóstico 0 se desarrollen debido a la restricción alimenticia indicada. La sobrealimentación puede influir en la alteración de los parámetros bioquímicos a controlar en la DM y HTA, control glicémico y presión arterial, y por lo tanto en las complicaciones asociadas a la desregulación de estos.

Es importante contar con instrumentos que faciliten la evaluación de variables psicosociales involucradas en el manejo y pronóstico de la enfermedad crónica. En la actualidad, existen cuestionarios que evalúan aspectos psicosociales por separado, tales como el afrontamiento de la enfermedad crónica (Welch, 1994; González, 2008), el apoyo social percibido (Revilla, Luna del Castillo, Bailón, \& Medina, 
2005), el estrés asociado a la enfermedad (Ortiz, Ortiz, Gatica, \& Gómez, 2011), la conducta alimentaria (Van Strien, Frijters, Bergers, \& Defares, 1986; Cepeda-Benito, Gleaves, Fernández, Vila, Williams, \& Reynoso, 2000; Castro, Bellido, \& Pertega, 2010; Pino, Díaz, y López, 2011), la percepción de la enfermedad (Moss-Morris et al, 2002), el manejo de la enfermedad (Schapira et al, 2012; Schmitt et al, 2013) y la calidad de vida asociada a ésta (Robles, Cortázar, Sánchez-Sosa, Paez, \& Nicolini, 2003). Sin embargo, estos cuestionarios son relativamente extensos o sólo evalúan un aspecto psicosocial, por lo que toma mucho tiempo realizar una evaluación integral. Considerando que el promedio de duración de una consulta médica ambulatoria oscila entre 15 y 20 minutos (Outomuro \& Actis, 2013), sería beneficioso contar con un sólo instrumento que evalúe distintas variables de manera rápida y simple, que permita identificar posibles focos de intervención. El propósito de este trabajo fue construir y validar un cuestionario que evaluara estrategias de afrontamiento, red de apoyo y conductas de sobrealimentación, y que sea lo suficiente breve para poder ser utilizado en atención primaria.

Se construyó el Cuestionario psicosocial para personas con diabetes e hipertensión y se procedió a analizar sus propiedades psicométricas en individuos con DM y/o HTA. La muestra pertenecía a un Programa de Telemonitoreo de DM y HTA en el cual se les entrega a los participantes un glucómetro y/o tensiómetro, según la patología, que al chequear el parámetro envía inmediatamente el dato a una plataforma web, la cual es monitoreada continuamente por un equipo de salud. Gracias a esto, los datos del cuestionario pudieron ser asociados a datos médicos tanto informados como observables con respecto al cuidado de la patología, tales como los resultados de sus controles bioquímicos y adherencia efectiva al programa.

\section{MÉTODO}

\section{Construcción del Cuestionario}

Se llevó a cabo una revisión de los cuestionarios/escalas existentes sobre afrontamiento 0 adaptación a la enfermedad crónica, apoyo social percibido y conducta alimentaria. Se seleccionaron 25 ítems que evaluaban estrategias de afrontamiento, apoyo social y sobrealimentación. La selección se realizó eligiendo aquellos ítems que representaran en mayor medida el constructo a medir a partir de la literatura y relevancia en población con DM y/o HTA desde la perspectiva de los autores y un equipo de profesionales de la salud expertos en ambas patologías. Todos los ítems tenían un formato de respuesta tipo Likert de cinco puntos que iba de muy en desacuerdo a muy de acuerdo o de nunca a siempre.

De afrontamiento de resignación pasiva se seleccionaron cuatro ítems correspondientes a la subescala con el mismo nombre del Diabetes Coping Measure (DCM) (Welch, 1994). De afrontamiento evitativo se seleccionaron tres ítems de la subescala con el mismo nombre del DCM, más uno extraído de una Escala de Actitudes hacia la DM (Bail Pupko \& Azzollini, 2012). De afrontamiento activo un doctor en psicología y un profesional del área de la salud, ambos externos al estudio, seleccionaron cuatro ítems de un conjunto generado por los investigadores. Con respecto al apoyo social, se seleccionaron los seis ítems pertenecientes a la escala con el mismo nombre del Cuestionario MOS de Apoyo Social (Revilla et al, 2005), cuatro de la subescala instrumental y dos de la emocional. Sobre Impulsividad en la Alimentación/Alimentación Externa y Alimentación Emocional se seleccionaron cuatro ítems del Food Craving Questionnaire (Cepeda-Benito et al., 2000), dos del Dutch Eating Behavior Questionnaire (Van Strien et al, 1986) y uno del Cuestionario de hábitos alimentarios en 
pacientes con sobrepeso y obesidad (Castro, Bellido, \& Pertega, 2010) (Tabla 1).

Los ítems seleccionados que estaban en inglés fueron traducidos al español por dos personas independientes, ambos bilingües de lengua nativa español. Posteriormente se revisaron las traducciones para llegar a un consenso y adaptarlas al español de Chile. Los enunciados que hacían referencia a una enfermedad crónica en particular, por ejemplo "diabetes", fueron cambiados a "enfermedad crónica".

TABLA 1.

Ítems seleccionados para el cuestionario de riesgo psicosocial en diabetes e hipertensión.

\begin{tabular}{|c|c|c|}
\hline Dimensión & $\begin{array}{l}\text { Escala } \\
\text { (autor principal, año) }\end{array}$ & 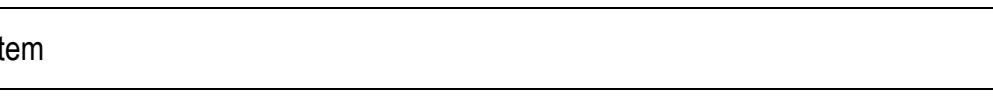 \\
\hline $\begin{array}{l}\text { Afrontamiento } \\
\text { Activo }\end{array}$ & Creación Propia & $\begin{array}{l}\text { - Tengo confianza en mis habilidades para manejar mi enfermedad crónica. } \\
\text { - Intento seguir las instrucciones del médico. } \\
\text { - Busco informarme sobre cómo cuidar mi salud. } \\
\text { - Invertir algo de tiempo y energía en el cuidado de mi enfermedad me } \\
\text { permite mantener el control de mi vida y salud. }\end{array}$ \\
\hline \multirow[t]{2}{*}{$\begin{array}{l}\text { Afrontamiento } \\
\text { Evitativo }\end{array}$} & $\begin{array}{l}\text { Diabetes Coping Measure } \\
\text { (Welch, 1994) }\end{array}$ & $\begin{array}{l}\text { - Me resisto a realizar mis controles cuando sé que no he cuidado bien mi } \\
\text { salud y por lo tanto mis resultados pueden salir mal. } \\
\text { - Es difícil integrar en mi ajetreada vida el chequeo diario de mi salud. } \\
\text { - No me gusta informarme sobre mi enfermedad debido a que sólo me } \\
\text { angustia más. }\end{array}$ \\
\hline & $\begin{array}{l}\text { Actitudes hacia la Diabetes } \\
\text { (Washington, 2009) }\end{array}$ & $\begin{array}{l}\text { - Creo que olvidarse de que uno padece una enfermedad es la mejor } \\
\text { manera de sentirse bien. }\end{array}$ \\
\hline $\begin{array}{l}\text { Afrontamiento } \\
\text { Resignación } \\
\text { Pasiva }\end{array}$ & $\begin{array}{l}\text { Diabetes Coping Measure } \\
\text { (Welch, 1994) }\end{array}$ & $\begin{array}{l}\text { - Siento que haga lo que haga, las complicaciones de mi enfermedad } \\
\text { crónica continuarán arruinando mi salud. } \\
\text { - Siento que me rindo ante mi enfermedad crónica. } \\
\text { - Siento que no puedo hacer mucho para mantener mi enfermedad crónica } \\
\text { controlada. } \\
\text { - Pareciera que siempre tengo mi enfermedad crónica desestabilizada sin } \\
\text { importar lo que haga. }\end{array}$ \\
\hline $\begin{array}{l}\text { Impulsividad en } \\
\text { la Alimentación/ } \\
\text { Alimentación } \\
\text { Externa }\end{array}$ & $\begin{array}{l}\text { Food Craving } \\
\text { Questionnaire - Trait } \\
\text { (Cepeda-Benito, 2000) }\end{array}$ & $\begin{array}{l}\text { - No tengo la fuerza de voluntad de resistir mis deseos de comer las } \\
\text { comidas que se me antojan. } \\
\text { - A veces, cuando como lo que se me antoja, pierdo control y como } \\
\text { demasiado. } \\
\text { - Una vez que me pongo a comer tengo problemas en dejar de comer. }\end{array}$ \\
\hline \multirow{3}{*}{$\begin{array}{l}\text { Alimentación } \\
\text { Emocional }\end{array}$} & $\begin{array}{l}\text { Cuestionario de hábitos } \\
\text { alimentarios para } \\
\text { pacientes con sobrepeso y } \\
\text { obesidad (Pino, 2011) }\end{array}$ & $\begin{array}{l}\text { - Si me dan ganas de comer a deshoras (entre comidas) no me puedo } \\
\text { controlar. }\end{array}$ \\
\hline & $\begin{array}{l}\text { Food Craving } \\
\text { Questionnaire - Trait } \\
\text { (Cepeda-Benito, 2000) } \\
\end{array}$ & - Comer me calma. \\
\hline & $\begin{array}{l}\text { Dutch Eating Behavior } \\
\text { Questionnaire (Van Strien, } \\
1986 \text { ) }\end{array}$ & $\begin{array}{l}\text { - Siento deseos de comer cuando estoy de bajo ánimo. } \\
\text { - Siento deseos de comer cuando estoy ansioso. }\end{array}$ \\
\hline $\begin{array}{l}\text { Apoyo Social } \\
\text { Instrumental }\end{array}$ & \multirow[t]{2}{*}{$\begin{array}{l}\text { Cuestionario de Apoyo } \\
\text { Social MOS (Revilla, 2005) }\end{array}$} & $\begin{array}{l}\text { - Alguien que le ayude cuando tenga que estar en la cama. } \\
\text { - Alguien que le lleve al médico cuando lo necesita. } \\
\text { - Alguien que le prepare la comida si no puede hacerlo. } \\
\text { - Alguien que le ayude en sus tareas domésticas si está enfermo. }\end{array}$ \\
\hline $\begin{array}{l}\text { Apoyo Social } \\
\text { Emocional }\end{array}$ & & $\begin{array}{l}\text { - Alguien con quien pueda contar cuando necesita hablar. } \\
\text { - Alguien en quien confiar o con quien hablar de sí mismo y sus } \\
\text { preocupaciones. }\end{array}$ \\
\hline
\end{tabular}


Previo al proceso de estudio de las propiedades psicométricas se llevó a cabo un piloto con 20 adultos (12 mujeres) diagnosticados con DM tipo 2 y/o HTA para evaluar la comprensión de las preguntas. Se observó una alta tasa de respuesta por ítem y todos los ítems puntuaron el nivel mínimo y máximo posible. Los participantes no reportaron dificultades de comprensión.

Estudio de propiedades psicométricas del cuestionario

\section{Participantes}

Doscientos sesenta y nueve adultos chilenos previamente diagnosticados con DM tipo 2 y/o HTA participaron voluntariamente en el estudio. Cincuenta y tres fueron excluidos por: presentar comorbilidad con otra enfermedad crónica (22), presentar un diagnóstico de depresión (4), dejar más de 3 ítems sin respuesta (4), no presentar estudios primarios completos (8) o haber rechazado participar en el programa de telemonitoreo, por lo que no se tenía acceso a sus datos sociodemográficos y médicos (15). La muestra final contaba con 216 participantes. Del total, 124 pertenecían al sistema de salud público (45 mujeres) y 92 al sistema privado (57 mujeres). El 16.7\% tenía DM, 29.6\% HTA y $53.7 \%$ DM y HTA. El promedio de edad era de 53.6 años ( $D E=10.2$; rango $=20-83$ ). El $28.4 \%$ presentaban educación escolar incompleta, $33.2 \%$ educación escolar completa y $38.4 \%$ educación superior. El $50.2 \%$ vivía con pareja e hijos, $23.9 \%$ vivía sólo con pareja, $12.4 \%$ vivía sólo con hijos, $4.3 \%$ vivía con sus padres, $1.9 \%$ vivía con otros familiares y $7.2 \%$ vivía solo. El $65.4 \%$ eran trabajadores remunerados y el $14.8 \%$ estaba jubilado.

\section{Variables Médicas}

Hemoglobina glicosilada. La concentración de hemoglobina glicosilada ( $\mathrm{HbA1c}$ ) en la sangre es un indicador de la glicemia de los últimos 2 a 4 meses. Altos valores de $\mathrm{HbA1C}$ indican un bajo nivel de control metabólico. Los participantes reportaron al momento de la evaluación su último valor obtenido.

Porcentaje de normo-glicemia. Porcentaje de controles con glicemias con valores normales (menores a $200 \mathrm{mg} / \mathrm{dl}$ ).

Porcentaje de normo-tensión. Porcentaje de controles con presiones arteriales con valores normales (sistólica menor a $170 \mathrm{mmHg} /$ diastólica menor a $100 \mathrm{mmHg}$ ).

Los porcentajes de normo-glicemia $y$ normo-tensión fueron calculados a partir de los datos de glicemia y presión arterial obtenidos por los participantes, con el glucómetro y tensiómetro entregados para realizar sus chequeos, durante los primeros 15 días del programa de telemonitoreo. A aquellos pacientes que presentan DM o DM y HTA, se les solicita realizar tres chequeos diarios, y aquellos que presentan sólo HTA se les solicita realizar dos chequeos diarios.

Adherencia al programa de telemonitoreo. Porcentaje de chequeos de glicemia y/o presión arterial realizados por el participante los primeros 15 días en el programa, con respecto a la frecuencia de chequeos solicitado según su(s) patología(s).

Adherencia a los medicamentos mediante Test MGL (Morisky, Green \& Levine, 1986). Evalúa la adherencia a los medicamentos mediante cuatro preguntas (sí o no) sobre actitud y conducta asociada a la toma de medicamentos. El puntaje total va de 0 a 4 . Para mejorar la comprensión, se invierten los puntajes de manera que puntajes más altos indican mayor adherencia a los medicamentos.

Índice de masa corporal (IMC). Razón (peso/estatura) ${ }^{2}$ calculado al momento del estudio.

Tabaquismo. Presencia o ausencia del hábito de fumar tabaco. 
Asistencia a Servicio de Urgencia. Asistencia o no al Servicio de Urgencia durante el mes anterior al estudio.

\section{Procedimiento}

Los participantes respondieron el cuestionario al momento de a una inducción para ingresar a un Programa de Telemonitoreo de su DM y/o HTA. En el caso de los participantes pertenecientes al sistema de salud público, esto se llevó a cabo en su centro de salud correspondiente y para los participantes del sistema privado se realiza en una sucursal de la institución a la que se encontraban afiliados. Se les informa a los participantes los objetivos y procedimiento del estudio. La participación fue voluntaria. La administración del cuestionario fue individual con un tiempo de duración de 15 minutos aproximadamente. Las variables peso y estatura (para cálculo de $I M C), H b A 1$, Test MGL, tabaquismo y asistencia a Servicio de Urgencia fueron recogidas mediante evaluación presencial con un profesional de la salud. Los datos de glicemia, presión arterial y adherencia al programa de telemonitoreo fueron obtenidos a través de la plataforma web que registra a distancia los chequeos realizados por los participantes.

\section{Análisis}

Para evaluar la validez de constructo, se procedió a realizar un análisis paralelo (Horn, 1965), seguido de un análisis factorial con método de ejes principales y rotación Varimax fijado en el número de factores obtenido en el análisis paralelo. Se estimó la fiabilidad del instrumento por medio del cálculo del índice de consistencia interna alfa de Cronbach. Para los siguientes análisis se calcularon los puntajes para cada factor a partir del promedio de los puntajes obtenidos en los reactivos. Se realizan correlaciones entre los factores para determinar sus asociaciones. Para la validez de criterio, se realizan regresiones múltiples y regresiones logísticas, según la naturaleza de la variable, con los factores como predictores y cada una de las variables médicas como variable dependiente. La regresión múltiple se realiza con método Stepwise y la regresión logística con método Enter. Todos los datos fueron analizados con el procesador estadístico SPSS 20, excepto el análisis paralelo que se llevó a cabo con el programa estadístico $\mathrm{R}$.

\section{RESULTADOS}

\section{Validez de Constructo}

Para la presente investigación, se procedió a realizar un Análisis Factorial con el objetivo de establecer la estructura del cuestionario. El test de esfericidad de Bartlett $\left(x^{2}=1755, p<.01\right)$ y la medida de Kaiser-Meyer-Olkin (.788), corroboraron la idoneidad de la matriz de correlaciones entre ítems para llevar a cabo el análisis factorial. El número de factores a extraer fue determinado por un análisis paralelo que indicó la presencia de 5 factores. Luego, los 25 ítems del cuestionario fueron sometidos a un primer análisis factorial según el método de ejes principales, con rotación Varimax fijado para 5 factores. Para la asignación de cada uno de los ítems a los factores se tuvo en cuenta la saturación de cada ítem en cada uno de los cinco factores. Los criterios de asignación fueron una saturación igual o superior a .352 según la fórmula de Stevens que incorpora el tamaño de la muestra (Stevens, 1986) y la coherencia teórica del ítem con el contenido del factor (teniendo en cuenta los demás ítems asignados al factor).

Dos ítems presentaron cargas sobre .352 en dos factores; "Creo que olvidarse de que uno padece una enfermedad es la mejor manera de sentirse bien" y "A veces, cuando como lo que se me antoja, pierdo control y como demasiado". Se procede a eliminar ambos ítems y a repetir los análisis descritos. Se mantienen buenos niveles de factorización. El análisis paralelo vuelve a indicar la existencia de 5 factores. Se realiza un segundo análisis factorial de ejes 
principales con rotación Varimax fijada en 5 dimensiones (Tabla 2). El primer factor fue nombrado "Apoyo Social" e incluye los ítems correspondientes a apoyo instrumental y emocional. El segundo ítem fue nombrado "Sobrealimentación" e incluye los ítems correspondientes a alimentación emocional y externa. Los factores 3,4 y 5 fueron nombrados "Afrontamiento Resignación Pasiva", "Afrontamiento Evitatito" y "Afrontamiento Activo", respectivamente.

TABLA 2.

Factores del cuestionario, con indicación de los pesos factoriales (método ejes principales con rotación varimax).

\begin{tabular}{|c|c|c|c|c|c|}
\hline \multirow{2}{*}{ Ítem cuestionario } & \multirow{2}{*}{$\mathrm{F} 1$} & \multirow[b]{2}{*}{$\mathrm{F} 2$} & \multirow[b]{2}{*}{ F3 } & \multirow[b]{2}{*}{ F4 } & \multirow[b]{2}{*}{ F5 } \\
\hline & & & & & \\
\hline Alguien que le ayude al estar en cama & .834 & & & & \\
\hline Alguien que le prepare la comida & .832 & & & & \\
\hline Alguien que ayude en tareas domésticas & .819 & & & & \\
\hline Alguien que le lleve al médico & .785 & & & & \\
\hline Alguien con quien pueda hablar & .748 & & & & \\
\hline Alguien en quien confiar & .701 & & & & \\
\hline Como cuando estoy ansioso & & .695 & & & \\
\hline Problemas en dejar de comer & & .661 & & & \\
\hline Comer me calma & & .579 & & & \\
\hline Como cuando estoy de bajo ánimo & & .565 & & & \\
\hline Con ganas de comer no me puedo controlar & & .527 & & & \\
\hline No tengo fuerza de voluntad & & .486 & & & \\
\hline Desestabilizada sin importar lo que haga & & & .670 & & \\
\hline Complicaciones continuarán & & & .637 & .334 & \\
\hline No puedo hacer mucho & & & .586 & & \\
\hline Siento que me rindo & & & .385 & & \\
\hline Es difícil integrar el chequeo & & & & .639 & \\
\hline No me gusta informarme & & & & .602 & \\
\hline Me resisto a realizar mis controles & & & & .532 & \\
\hline Busco informarme & & & & & .652 \\
\hline Tengo confianza en mis habilidades & & & & & .544 \\
\hline Intento seguir las instrucciones del médico & & & & & .461 \\
\hline Invertir tiempo y energía mantiene el control & & & & & .373 \\
\hline
\end{tabular}

En cuanto a las correlaciones entre factores (Tabla 3), los resultados muestran que existe una alta coherencia entre los que se refieren al mismo constructo. En particular, Afrontamiento Activo correlaciona negativamente con Afrontamiento Evitativo $(r=-.158, p<.05)$ y Afrontamiento de Resignación Pasiva ( $r=-.200, p<.01)$, que correlacionan positivamente entre sí $(r=$ $.444, p<.01$ ). Esto confirma que los estilos de afrontamiento evitativo y de resignación pasiva tienden a coexistir y asociarse de 
manera negativa con el afrontamiento activo.

Por otra parte, los resultados confirman relaciones entre los distintos constructos. Apoyo Social correlaciona de manera positiva con Afrontamiento Activo ( $\mathrm{r}$ $=.210, p<.01$ ), mientras que correlaciona negativamente con Afrontamiento Evitativo $(r=-.159, p<.05)$, Afrontamiento de Resignación Pasiva $(r=-.139, p<.05)$ y Sobrealimentación $(r=-.192, p<.05)$, mostrando que la percepción del apoyo social se asocia a las estrategias de afrontamiento utilizadas al lidiar con la enfermedad crónica y a episodios de sobrealimentación. Por otro lado, Sobrealimentación también se asocia a los tres tipos de afrontamiento, correlacionando negativamente con Afrontamiento Activo ( $r=$ $-.158, p<.05)$ y positivamente con Afrontamiento Evitativo $(r=.164, p<.05)$ y Afrontamiento de Resignación Pasiva $(r=$ $.333, p<.01$ ). Esto sugeriría que quienes utilizan en mayor medida estrategias de afrontamiento evitativas y/o de resignación pasiva presentarían en mayor frecuencia episodios de alimentación emocional y/o impulsividad asociada a la alimentación.

\section{Análisis de Consistencia Interna}

Una vez conformados los factores, se obtuvo el Alfa de Cronbach para cada uno de ellos (Tabla 3). Los factores Apoyo Social, Afrontamiento de Resignación y Sobrealimentación presentaron una buena consistencia interna, con valores de .915, .727 y .774 , respectivamente. El factor de Afrontamiento Evitativo presentó un nivel de consistencia interna es más débil, de 690 . Finalmente, el factor Afrontamiento Activo presentó una consistencia interna baja, con un valor de .547. Una de las razones posibles para los bajos niveles de consistencia interna de los factores de Afrontamiento Evitativo y Afrontamiento Activo puede ser el bajo número de ítems que los componen (3).

TABLA 3.

Medias, desviaciones estándar, correlaciones y coeficientes de alfa de cronbach de las subescalas.

\begin{tabular}{llllllll}
\hline & Media & DE & 1 & 2 & 3 & 4 & 5 \\
\hline 1. Afrontamiento Activo & 4.22 & .68 & $(.547)$ & & & & \\
2. Afrontamiento Evitativo & 2.31 & 1.14 & $-.150^{*}$ & $(.690)$ & & & \\
3. Afrontamiento de Resignación Pasiva & 2.44 & 1.01 & $-.200^{* *}$ & $.444^{* *}$ & $(.727)$ & & \\
4. Sobrealimentación & 2.73 & .93 & $-.158^{*}$ & $.164^{*}$ & $.333^{* *}$ & $(.774)$ & \\
5. Apoyo Social & 3.96 & 1.06 & $.210^{* *}$ & $-.159^{*}$ & $-.139^{*}$ & $-.192^{* *}$ & $(.915)$ \\
\hline Nota. Entre paréntesis se indican los coeficientes alfa correspondientes a las subescalas. & & & & \\
${ }^{*} p<.05,{ }^{* *} p<.01$. & & & & & &
\end{tabular}

Validez de Criterio

Se llevó a cabo un análisis de regresión múltiple con los factores como variables predictoras y cada una de las variables médicas continuas como variable dependiente, para estimar el grado en que cada subescala o factor contribuye a la variable médica. Para las variables médicas dicotómicas se realiza el mismo proceso con regresión logística (Tabla 4).
La adherencia al programa es explicada de manera significativa por el Afrontamiento Evitativo ( $B=-.403, p<.01)$. No obstante, se observa una tendencia a ser explicada también por el Afrontamiento Activo ( $\mathrm{B}=$ $.144, p=.05)$. La adherencia a los medicamentos es predicha de manera significativa por el Afrontamiento Evitativo ( $B$ $=-.169, p<.01) y$ por el Afrontamiento Activo $(B=.443, p<.01)$. El Afrontamiento Activo aparece como predictor único del 
IMC $(B=-1.752, p<.01)$ y de la presencia de tabaquismo $(B=-.731, p<.01)$.

El Afrontamiento de Resignación Pasiva se observa como predictor único significativo para $\mathrm{HbA1c}(\mathrm{B}=.955, \mathrm{p}<.01)$, para el porcentaje de normoglicemia $(B=$ $11.335, p<.01$ ), para el porcentaje de normopresión $(B=-2.793, p<.05)$ y para asistencia al Servicio de Urgencias $(B=$ $.723, p<.05)$.

Por lo tanto, la mayor utilización de estrategias de afrontamiento activas y la menor utilización de estrategias de afrontamiento evitativas, estaría asociado a una mayor adherencia a los tratamientos e indicadores de salud a largo plazo como es el IMC. Con respecto al hábito de fumar, el menor uso de estrategias de afrontamiento activas, aumentaría las probabilidades de que la persona incurra en esta conducta. Por otro lado, la utilización de estrategias de resignación pasiva estaría asociado a mayores descompensaciones de la enfermedad crónica.

Los factores Apoyo Social y Sobrealimentación no presentaron coeficientes significativos en ninguna de las variables médicas.

TABLA 4.

Análisis de regresión múltiple y logística con los factores como predictores y las variables médicas como variables dependientes.

\begin{tabular}{|c|c|c|c|c|c|c|}
\hline Variable dependiente & Predictor(es) & B & $t$ & $\mathrm{p}$ & $\mathrm{F}$ & $\mathrm{R}^{2}$ \\
\hline Adh. programa & A. Evitativo & -.403 & -2.901 & .004 & $8.416^{\star *}$ & .044 \\
\hline \multirow[t]{2}{*}{ Adh. medicamentos } & A. Activo & .443 & 4.166 & .000 & $14.291^{* *}$ & .126 \\
\hline & A. Evitativo & -.169 & -2.631 & .009 & & \\
\hline IMC & A. Activo & -1.752 & -3.208 & .002 & $10.292^{* *}$ & .047 \\
\hline $\mathrm{HbA} 1$ & A. Resignación Pasiva & .955 & 2.949 & .000 & $8.696^{* *}$ & .179 \\
\hline$\%$ normoglicemia & A. Resignación Pasiva & -11.335 & -3.866 & .000 & $14.949^{* *}$ & .095 \\
\hline \multirow[t]{2}{*}{ \% normotensión } & A. Resignación Pasiva & -2.793 & -2.191 & .030 & $4.802^{*}$ & .030 \\
\hline & & $B$ & OR & $p$ & $x^{2}$ & $\begin{array}{l}-2 \log \\
\text { likelihood }\end{array}$ \\
\hline Asistencia a Urgencias & A. Resignación Pasiva & .723 & 2.060 & .020 & $13.966^{*}$ & 96.868 \\
\hline Tabaquismo & A. Activo & -.731 & .481 & .002 & $19.912^{* *}$ & 260.913 \\
\hline
\end{tabular}

\section{DISCUSIÓN}

El objetivo de este estudio fue construir un cuestionario que evaluara aspectos psicosociales que tienen implicancia en el manejo de enfermedades crónicas y evaluar sus propiedades psicométricas. A partir de un conjunto de ítems tanto creados por los autores como obtenidos a través de una revisión de los cuestionarios existentes en español e inglés, se seleccionan 25 ítems que evalúan estrategias de afrontamiento de la enfermedad crónica, conducta alimentaria y apoyo social. La evaluación de sus propiedades psicométricas se llevó a cabo con una muestra formada por personas con el diagnóstico de diabetes mellitus tipo 2 y/o hipertensión arterial pertenecientes al sistema público y privado, que ingresaban a un programa de monitoreo a distancia de su enfermedad. Al respecto, se han obtenido indicadores aceptables de validez de constructo, fiabilidad y validez de criterio.

La validación de constructo del cuestionario redujo el número de ítems a 23. Se identificaron 5 factores correspondientes a: afrontamiento activo, afrontamiento evitativo, afrontamiento resignación pasiva, sobrealimentación y apoyo social. Las 
subescalas presentaron una fiabilidad de regular a excelente, a excepción de la subescala de afrontamiento activo. Las relaciones entre las subescalas del mismo constructo y entre constructos diferentes se muestran coherentes a partir de lo expuesto en la literatura.

Las subescalas de estrategias de afrontamiento se asocian a las variables médicas incluidas en el estudio que dicen relación con la gravedad de la patología y la adherencia a los regímenes de autocuidado. Una de las fortalezas del estudio fue contar con la posibilidad de obtener datos directamente observables de tanto el control de la enfermedad como la adherencia al tratamiento gracias a que los pacientes se encontraban en un programa de telemonitoreo. Cabe mencionar que, si bien los tres tipos de afrontamiento se asocian a las variables médicas, las estrategias de afrontamiento activo y de tipo evitativo se asociaron más a la adherencia a los tratamientos, mientras que las estrategias de afrontamiento de resignación pasiva se asociaron más a los niveles bioquímicos. Por lo tanto, el cuestionario resultaría útil como guía sobre los aspectos a intervenir según las estrategias de afrontamiento que utiliza el paciente.

Una limitación de este estudio es que no se compara la validez predictiva de las subescalas con la de las escalas más extensas de las cuales fueron extraídas, futuros estudios podrían realizar tal comparación con el fin de reforzar la importancia de la versión breve. Además, la no existencia de datos longitudinales no permite establecer la estabilidad temporal del instrumento (confiabilidad test-retest) y la no inclusión de pacientes con otras enfermedades crónicas o diabéticos tipo 1 limita su uso a población de diabéticos tipo 2 e hipertensos. El bajo indicador de fiabilidad de la subescala de afrontamiento activo (0.547), pese a su asociación con datos médicos (adherencia a medicamentos y programa, IMC, tabaquismo) sugiere mayor estudio de este último factor en futuras investigaciones. Asimismo, se propone que este estudio sea replicado con una muestra de pacientes crónicos de mayor tamaño, así como también de otras áreas geográficas, con la finalidad de aumentar la representatividad del estudio. Por otro lado, los participantes obtuvieron elevados puntajes en las subescalas de afrontamiento activo y apoyo social. Esto posiblemente se debe a lo siguiente: (1) los participantes aceptaron de manera voluntaria participar de un programa de monitoreo de su enfermedad, lo que sugeriría que se encontraban más activos en el cuidado de su patología, y (2) uno de los requisitos para ingresar al programa era presentar una red de apoyo que permitiese tener contacto con los profesionales de la salud del programa de ser necesario. Futuros estudios que contribuyan a la validación del cuestionario debiesen incluir pacientes que no se encuentren participando en un programa específico para el tratamiento de su enfermedad. En aquellos estudios se esperaría encontrar mayor variabilidad en el uso de las estrategias de afrontamiento y en el apoyo social percibido.

Por otro lado, llama la atención la baja asociación entre la conducta alimentaria, el apoyo social y las variables médicas. Particularmente llamativa es la falta de asociación entre sobrealimentación e IMC.

Pese a lo anterior, los resultados del estudio muestran que se construyó un cuestionario de fácil y rápida aplicación, que posee adecuadas propiedades psicométricas para su utilización en población de pacientes con diabetes tipo 2 y/o hipertensión. Altos puntajes en las subescalas de afrontamiento evitativo, afrontamiento de resignación pasiva y sobrealimentación 0 bajos puntajes en relación al apoyo social y afrontamiento activo podrían sugerir que la persona presenta cierto grado de descompensación de su patología y/o un bajo cuidado de su 
salud. Asimismo, ese perfil posiblemente presente malestar psicológico asociado a su patología, con riesgo de padecer 0 desarrollar un trastorno anímico. En particular, la identificación de subescalas permite la planificación de intervenciones específicas y su posterior evaluación, así como también su uso en estudios científicos que involucren a individuos diabéticos tipo 2 y/o hipertensos.

\section{REFERENCIAS}

Aldwin, C. M. (2007). Stress, coping, and development: An integrative perspective. Guilford Press.

Ary, D. V., Toobert, D., Wilson, W., \& Glasgow, R. E. (1986) Patient perspective on factors contributing to nonadherence to diabetes regimen. Diabetes Care, 9(2), 168-172. doi: 10.2337/diacare.9.2.168

Bail Pupko, V., \& Azzollini, S. (2012). Actitudes, afrontamiento y autocuidado en pacientes con diabetes tipo 2 . Revista Argentina de salud pública, 3(10), 15-23. Recuperado de: http://www.rasp.msal.gov.ar/rasp/articul os/

Bowen, K. S., Uchino, B. N., Birmingham, W., Carlisle, M., Smith, T. W., \& Light, K. C. (2014). The stress-buffering effects of functional social support on ambulatory blood pressure. Health Psychology, 33(11), 1440-1443. doi: 10.1037/hea0000005

Castro, P., Bellido, D., \& Pertega, S. (2010). Elaboración y validación de un nuevo cuestionario de hábitos alimentarios para pacientes con sobrepeso y obesidad. Endocrinología y nutrición, 57(4), 130-139. doi: 10.1016/j.endonu.2010.02.006

Cepeda-Benito, A., Gleaves, D. H., Fernández, M. C., Vila, J., Williams, T.
L., \& Reynoso, J. (2000). The development and validation of Spanish versions of the state and trait food cravings questionnaires. Behavior Research and Therapy, 38(11), 11251138. doi: 10.1016/s00057967(99)00141-2

Coffman, M. J.(2008). Effects of tangible social support and depression on diabetes self-efficacy. Journal of Gerontological Nursing, 34(4), 32-39. doi: 10.3928/00989134-20080401-02

Dolbier, C. L., \& Steinhardt, M. A. (2000). The development and validation of the Sense of Support Scale. Behavioral Medicine, 25(4), 169-179. doi: 10.1080/08964280009595746

Duangdao, K. M., \& Roesch, S. C. (2008). Coping with diabetes in adulthood: a meta-analysis. Journal of Behavioral Medicine, 31(4), 291-300. doi: 10.1007/s10865-008-9155-6

Feifel, H., Strack, S., \& Nagy, V. T. (1987). Coping strategies and associated features of medically ill patients. Psychosomatic medicine, 49(6), 616625. doi: 10.1097/00006842198711000-00007

FID (2015). Séptima edición del Atlas de la Diabetes de la Federación Internacional de la Diabetes. Recuperado de: http://www.diabetesatlas.org/

Franz, M. J., Bantle, J. P., Beebe, C. A., Brunzell, J. D., Chiasson, J.-L., Garg, A., ... Wheeler, M. (2002). EvidenceBased Nutrition Principles and Recommendations for the Treatment and Prevention of Diabetes and Related Complications. Diabetes Care, 25(1), 148-198. doi: 10.2337/diacare. 25.1.148

Gallant, M. P. (2003). The influence of social support on chronic illness selfmanagement: a review and directions for research. Health Education $y$ Behavior, 30(2), 170-195. doi: $10.1177 / 1090198102251030$

González, M. T. (2008). Análisis de un instrumento de evaluación del 
afrontamiento del estrés en sujetos con cáncer. Revista Iberoamericana de Diagnóstico y Evaluación - e Avaliação Psicológica, 25(1), 35-49. Recuperado de: http://www.aidep.org/

Hall, R. F., Joseph, D. H. \& SchwartzBarcott, D. (2003) Overcoming obstacles to behavior change in diabetes self-management. Diabetes Educator, 29(2), 303-11. doi: 10.1177/014572170302900221

Horn, J. L. (1965). A rationale and test for the number of factors in factor analysis. Psychometrika, 30(2), 179-185. doi:10.1007/bf02289447

Iturralde, E., Weissberg-Benchell, J., \& Hood, K. K. (2016). Avoidant Coping and Diabetes-Related Distress: Pathways to Adolescents' Type 1 Diabetes Outcomes. Health Psychology. doi:10.1037/hea0000445

Lazarus, R. S., \& Folkman, S. (1984). Coping and adaptation. The handbook of behavioral medicine, 282-325.

Nguyen, H., Odelola, O. A., Rangaswami, J., \& Amanullah, A. (2013). A Review of Nutritional Factors in Hypertension Management. International Journal of Hypertension, 2013, 1-12. doi: $10.1155 / 2013 / 698940$

Manne, S. (2001) Chronic illness, psychosocial coping with. International Encyclopedia of the Social and Behavioral Sciences, 1773-1779. doi: 10.1016/B0-08-043076-7/03809-2

Marín, F., \& Rodríguez, M. (2001). Apoyo familiar en el apego al tratamiento de la hipertensión arterial esencial. Salud Pública de México, 43(4), 336-339. doi: org/10.1590/s003636342001000400010

Menéndez, C., Montes, A., Gamarra, T., Núñez, C. N., Fachado, A. \& Bujan, S. (2003). Influencia del apoyo social en pacientes con hipertensión arterial esencial. Atención primaria, 31(8), 506513. doi: $10.1157 / 13047733$

MINSAL (2010). Encuesta Nacional de Salud 2009-2010. Recuperado de: http://web.minsal.cl
MINSAL (2011). Metas 2011 - 2020. ESTRATEGIA NACIONAL DE SALUD Para el cumplimiento de los Objetivos Sanitarios de la Década 2011-2020. Recuperado de: http://web.minsal.cl

Morisky, D. E., Green, L. W., \& Levine, D. M. (1986). Concurrent and predictive validity of a self-reported measure of medication adherence. Medical care, 24(1), 67-74. doi: 10.1097/00005650198601000-00007

Moorey, S., y Greer, S. (1989). Psychological therapy for patients with cancer: a new approach. American Psychiatric Publishing, Inc.

Moss-Morris, R., Weinman, J., Petrie, K., Horne, R., Cameron, L., \& Buick, D. (2002). The revised illness perception questionnaire (IPQ-R). Psychology and health, 17(1), 1-16. doi: 10.1037/t11973-000

Organización Mundial de la Salud. (2014). Informe sobre la situación mundial de las enfermedades no transmisibles 2014. Recuperado de: www.oms.cl

Ortiz, M., Ortiz, E., Gatica, A., \& Gómez, D. (2011). Factores psicosociales asociados a la adherencia al tratamiento de la Diabetes Mellitus tipo 2. Terapia psicológica, 29(1), 5-11. doi: doi.org/10.4067/s071848082011000100001

Outomuro, D., \& Actis, A. M. (2013). Estimación del tiempo de consulta ambulatoria en clínica médica. Revista médica de Chile, 141(3), 361-366. doi: 10.4067/S0034-98872013000300012

Ouwens, M. A., van Strien, T., \& van Leeuwe, J. F. (2009). Possible pathways between depression, emotional and external eating. A structural equation model. Appetite, 53(2), 245-248. doi: 10.1016/j.appet.2009.06.001

Peyrot, M., Rubin, R. R., Lauritzen, T., Snoek, F. J., Matthews, D. R., \& Skovlund, S. E. (2005). Psychosocial problems and barriers to improved diabetes management: results of the Cross-National Diabetes Attitudes, 
Wishes and Needs (DAWN) Study. Diabetic Medicine, 22(10), 1379-1385. doi: 10.1111/j.1464-5491.2005.01644.x

Pino, J. L., Díaz, C., \& López, M. Á. (2011). Construcción y validación de un cuestionario para medir conductas y hábitos alimentarios en usuarios de la atención primaria de salud. Revista chilena de nutrición, 38(1), 41-51. doi: 10.4067/s0717-75182011000100005

Racine, S. E., Culbert, K. M., Larson, C. L., \& Klump, K. L. (2009). The possible influence of impulsivity and dietary restraint on associations between serotonin genes and binge eating. Journal of Psychiatric Research, 43(16), 1278-1286. doi: doi.org/10.1016/j.jpsychires.2009.05.00 2

Revilla, L., Luna del Castillo, J., Bailón, E., \& Medina, I. (2005). Validación del cuestionario MOS de apoyo social en Atención Primaria. Medicina de Familia, 6(1), 10-18. Recuperado de: http://www.samfyc.es/Revista

Robles. R., Cortázar, J., Sánchez-Sosa, J., Paez, F., \& Nicolini H. (2003). Evaluación de la calidad de vida en Diabetes Mellitus Tipo II: propiedades psicométricas de la versión en español del DQOL. Psicothema, 15(2), 247-252. Recuperado de: http://www.psicothema.com

Rodríguez, S., \& Enrique, H. (2007). Validación Argentina del cuestionario MOS de apoyo social percibido. Psicodebate, 7(7), 155-168. doi: 10.18682/pd.v7i0.433

Schapira, M. M., Fletcher, K. E., Hayes, A., Eastwood, D., Patterson, L., Ertl, K., \& Whittle, J. (2012). The development and validation of the hypertension evaluation of lifestyle and management knowledge scale. The Journal of Clinical Hypertension, 14(7), 461-466. doi: 10.1111/j.1751-7176.2012.00619.x

Schmitt, A., Gahr, A., Hermanns, N., Kulzer, B., Huber, J., \& Haak, T. (2013). Instrument to assess diabetes self-care activities associated with glycaemic control. Health and Quality of Life Outcomes, 11(1), 138 . doi: 10.1186/1477-7525-11-138

Shah, B. M., Gupchup, G. V., Borrego, M. E., Raisch, D. W., \& Knapp, K. K. (2012). Depressive symptoms in patients with type 2 diabetes mellitus: do stress and coping matter? Stress and Health, 28(2), 111-122. doi: 10.1002/smi. 1410

Siqueira, P. D., Dos Santos, M. A., Zanetti, M. L., \& Ferronato, A. A. (2007). Dificultades de los pacientes diabéticos para el control de la enfermedad: sentimientos y comportamientos. Revista Latino-Americana de Enfermagem, 15(6), 1105-12. Recuperado de: http://www.scielo.br

Solís, C.L., Aguirre, M.L., Godorecci, S., Mois, P., Rojas, H., Jiménez, R. (2008). Prevalencia de Diabetes mellitus en Chile. Revista ALAD, 16(3), 93-97. Recuperado de: http://www.revistaalad.com

Stanton, A. L., Revenson, T. A., \& Tennen, H. (2007). Health psychology: psychological adjustment to chronic disease. Annual Review of Psychology, 58(1), 565-592. doi: 10.1146/annurev.psych.58.110405.085 615

Stevens J. (1986) Power of the multivariate analysis of variance tests. Psychological Bulletin, 88(3), 728-737. doi:10.1037/0033-2909.88.3.728

Spoor, S. T., Bekker, M. H., Van Strien, T., \& van Heck, G. L. (2007). Relations between negative affect, coping, and emotional eating. Appetite, 48(3), 368376. doi: 10.1016/j.appet.2006.10.005

Svensson, T., Inoue, M., Sawada, N., Yamagishi, K., Charvat, H., Saito, I., ... \& Mimura, M. (2016). Coping strategies and risk of cardiovascular disease incidence and mortality: the Japan Public Health Center-based prospective Study. European heart journal, 37(11), 890-899.

doi: 
Turan, B., Osar, Z., Turan, J. M., Damci, T., \& Ilkova, H. (2008). The role of coping with disease in adherence to treatment regimen and disease control in type 1 and insulin treated type 2 diabetes mellitus. Diabetes \& Metabolism, 28(3), 186-193. doi: DM-06-2002-28-3-12623636-101019-ART3

Van Strien, T., Frijters, J. E., Bergers, G., \& Defares, P. B. (1986). The Dutch Eating Behavior Questionnaire (DEBQ) for assessment of restrained, emotional, and external eating behavior. International Journal of Eating Disorders, 5(2), 295-315. doi: 10.1002/1098-

$108 \times(198602) 5: 2<295::$ aideat2260050209>3.0.co;2-t

Vega, O. M., \& González, D. S. (2009). Apoyo social: elemento clave en el afrontamiento de la enfermedad crónica. Enfermería Global, (16), 1-15. doi: $\quad 10.4321 / \mathrm{s} 1695$ 61412009000200021

Welch, G. (1994). The Diabetes Coping Measure: $A$ measure of cognitive and behavioral coping specific to diabetes. Handbook psychology and diabetes: A guide to psychological measurement in diabetes research and practice.

Westaway, M. S., Seager, J. R., Rheeder, P. \& Van Zyl, D. G. (2005). The effects of social support on health, well-being and management of Diabetes Mellitus: A black South African perspective. Ethnicity \& Health, 10(1), 73-89. doi: 10.1080/135578505200032304

Yannakoulia, M. (2006) Eating behavior among type 2 diabetic patients: A poorly recognized aspect in a poorly controlled disease. The Review of Diabetic Studies, 3(1), 11-16. doi: 10.1900/rds.2006.3.11 